\title{
Three new species of Conlarium from sugarcane rhizosphere in southern China
}

\author{
Ling Xie ${ }^{1,2^{*}}$, Yan-Lu Chen ${ }^{1,2^{*}}$, Yan-Yan Long', Yan Zhang', \\ Shi-Tong Liao', Bin Liu², Li-Ping Qin', Qian Nong', Wen-Long Zhang' \\ I Microbiology Research Institute, Guangxi Academy of Agricultural Science, Nanning, Guangxi Province \\ 530007, China 2 Institute of Applied Microbiology, Agricultural College of Guangxi University, Nanning, \\ Guangxi Province 530005, China \\ Corresponding author: Wen-Long Zhang (lon1933@163.com)
}

Academic editor: Cecile Gueidan | Received 9 May 2019 | Accepted 20 June 2019 | Published 5 July 2019

Citation: Xie L, Chen Y-L, Long Y-Y, Zhang Y, Liao S-T, Liu B, Qin L-P, Nong Q, Zhang W-L (2019) Three new species of Conlarium from sugarcane rhizosphere in southern China. MycoKeys 56: 1-11. https://doi.org/10.3897/ mycokeys. 56.35857

\begin{abstract}
Three new species isolated from sugarcane rhizosphere in China, namely Conlarium baiseense sp. nov., $C$. nanningense sp. nov., and $C$. sacchari sp. nov., are described and illustrated. Molecular evidence (phylogenetic analysis of combined LSU, SSU, ITS and RPB2 sequence data) and phenotypical characters support their independent status from related and similar species. The new species, as dark spetate endophytes, inhabit sugarcane rhizosphere and can form a symbiosis with sugarcane.
\end{abstract}

\section{Keywords}

Conlariaceae, conidial fungi, phylogeny, Rhizosphere, taxonomy

\section{Introduction}

The genus Conlarium, described by Liu et al. (2012), belongs to the Conlariaceae, a family of freshwater ascomycetes (Zhang et al. 2017). This genus includes three species: C. duplumascospora, C. aquaticum, and C. thailandense. In these species, C. duplumascospora and C. aquaticum were isolated from submerged woody samples in streams (Liu et al. 2012; Zhang et al. 2017) and C. thailandense was isolated from dead wood (Phookamsak et al. 2019). During our ongoing survey of dark septate endophytes which inhabit sugarcane rhizosphere in Guangxi province, China, three undescribed

* co-first authorship

Copyright Ling Xie et al. This is an open access article distributed under the terms of the Creative Commons Attribution License (CC BY 4.0), which permits unrestricted use, distribution, and reproduction in any medium, provided the original author and source are credited. 
species with the morphological characteristics of the genus Conlarium were isolated by the baiting method. The specimens were deposited in the Mycological Herbarium, Institute of Microbiology, Chinese Academy of Sciences, Beijing, China (HMAS).

\section{Materials and methods}

\section{Fungal isolation and morphological studies}

All soil samples were collected from the $5-15 \mathrm{~cm}$ deep sugarcane rhizosphere by five sampling methods in Guangxi province, China. Fungal isolations were obtained by using Chinese cabbage as a baiting plant, as described by Narisawa et al. (1998). Cultural characteristics were recorded after two weeks from potato dextrose agar (PDA). Conidiophores, conidiogenous cells, and conidia were examined as slide fungal preparations mounted in PVLG (polyvinyl alcohol, Lactic acid, Glycerin, and MiliQ water). Observations and measurements were made with Olympus BX53 Ci-L light microscope. Scanning electron microscopy (SEM) used a Tescan-vega3 LMU SEM.

\section{Molecular sequencing and phylogenetic analysis}

The genomic DNA was extracted from mycelium grown on PDB (potato dextrose broth) at $28{ }^{\circ} \mathrm{C}$ for $10 \mathrm{~d}$ using the Prepman Ultra Sample Preparation Reagent Protocol (Applied Biosystems, California, USA). The large subunit ribosomal RNA gene (LSU), the small subunit ribosomal RNA gene (SSU), the internal transcribed spacer (ITS) rDNA, and the RNA polymerase II subunit 2 (RPB2) were amplified with fungal specific primers LROR/LR5, NS1/NS4, ITS1/ITS4, and fRPB2-5f/fRPB2-7cR (Vilgalys and Hester 1990; White et al. 1990; Liu et al. 1999). The PCR reaction mixture and conditions followed the modified protocol of $2 \times$ EasyTaq PCR SuperMix (TransGen Biotech, Beijing, China). Amplification was performed in a $50 \mu \mathrm{L}$ reaction volume which contained PCR buffer $\left[20 \mathrm{mM} \mathrm{KCl,} 10 \mathrm{mM}\left(\mathrm{NH}_{4}\right)_{2} \mathrm{SO}_{4}, 2 \mathrm{mM}\right.$ $\mathrm{MgCl}_{2}, 20 \mathrm{mM}$ Tris-HCl, pH8.4], $200 \mu \mathrm{M}$ of each deoxyri-bonucleotide triphosphate, 15 pmols of each primer, $100 \mathrm{ng}$ template DNA, and 2.5 units of Taq DNA polymerase (Biocolor BioScience and Technology, Shanghai, China). The thermal cycling program was as follows: 5 min initial denaturation at $94^{\circ} \mathrm{C}$, followed by 35 cycles of $40 \mathrm{~s}$ denaturation at $94^{\circ} \mathrm{C}, 40 \mathrm{~s}$ annealing at $56^{\circ} \mathrm{C}, 60 \mathrm{~s}$ extension at $72{ }^{\circ} \mathrm{C}$, and a final 10 min extension at $72^{\circ} \mathrm{C}$. A negative control using sterilized distilled water instead of template DNA was included in the amplification process. The PCR products were examined by electrophoresis at $75 \mathrm{~V}$ for $2 \mathrm{~h}$ in $0.8 \%$ (W/V) agarose gel in $1 \times \mathrm{TAE}$ buffer (0.4 M Tris, $50 \mathrm{mM} \mathrm{NaOAc,} 10 \mathrm{mM}$ EDTA, pH 7.8) and visualized under ultraviolet light after staining with ethidium bromide $\left(0.5 \mu \mathrm{g} \mathrm{ml}^{-1}\right)$. The PCR products were purified using PCR Cleanup Filter Plates (MultiScreen ${ }^{\circledR} \mathrm{PCR} \mu$ 96; Millipore, USA) according to the manufacturer's protocol. Purified PCR products were directly sequenced with primer pairs, as mentioned above, in an ABI 3730-XL DNA sequencer 
(Applied Biosystems, USA). The sequences were deposited at GenBank (http://www. ncbi.nlm.nih.gov) and compared in BLAST. Four kinds of rDNA sequences together with reference sequences (Table 1) were respectively aligned by MEGA v. 6.0 based on the neighbor-joining analyses and 1000 bootstrap replications.

Bayesian analyses of the same aligned four kinds of rDNA sequences dataset were conducted with MrBayes v. 3.1.2 (Huelsenbeck and Ronquist 2001) following the protocol of Sun and Guo (2010). The best-fit evolutionary model was determined for each dataset by comparing different evolutionary models via MrModeltest v. 2.3 (Nylander 2008). Four simultaneous chains of Markov Chain Monte Carlo were run starting from random trees and sampling every 100 generations. The analyses were halted at 4,000,000 generations for four kinds of rDNA sequences, when the calculation reached stationarity. At the end of the analysis, 4,000 trees were generated, respectively, and $25 \%$ of them were excluded as the "burn in" when calculating the posterior probabilities. Bayesian posterior probabilities were obtained from the $50 \%$ majority rule consensus trees that remained. If more than $95 \%$ of the sampled trees contained a given clade, it was considered to be significantly supported by our data.

\section{Results}

Taxonomy

Conlarium nanningense L.Xie, Y.L.Chen \& B.Liu, sp. nov. MycoBank: MB821416

Figure 1

Etymology. The species is named for Nanning City, the type locality.

Type. CHINA. Guangxi: Nanning City, Datang Town. $22^{\circ} 23^{\prime} 25^{\prime \prime N}, 108^{\circ} 23^{\prime} 12^{\prime \prime E}$, $144 \mathrm{~m}$ alt., in sugarcane rhizosphere, 11 Feb. 2011, L. Xie, M1 (HMAS 247075 holotype) deposited in Microbiology Research Institute, Guangxi Academy of Agricultural Science.

Description. Colony reached $22 \mathrm{~mm}$ diameter on PDA medium after 2 weeks, grey-white to grey-brown, nearly circular, flat growth, less aerial hyphae. Hyphae greybrown, verruculose, septate. Conidiophores $1-15 \times 1-5 \mu \mathrm{m}(6 \pm 3 \times 4 \pm 1 \mu \mathrm{m}, n=$ 54), stubby, unbranched, septate or aseptate, straight or flexuous, hyaline, becoming brown with age. Conidiogenous cells determinate, doliiform, cylindrical, 4-13 $\times 5-10$ $\mu \mathrm{m}(6 \pm 2 \times 7 \pm 2 \mu \mathrm{m}, n=22)$. Conidia brown, muriform, irregularly globose or subglobose, smooth, constricted at the septa, $0-1$ transversely septa, $0-4$ longitudinal septa, $11-21 \times 9-21 \mu \mathrm{m}(15 \pm 3 \times 13 \pm 3 \mu \mathrm{m}, n=50)$. Chlamydospores subglobose or irregular, $4-12 \mu \mathrm{m}(7 \pm 2 \mu \mathrm{m}, n=67)$. Sexual morph: undetermined.

Habitat and distribution. In sugarcane rhizosphere soil of southern China.

Other specimens examined. CHINA. Guangxi: Nanning City, Datang Town. $22^{\circ} 29^{\prime} 54.51^{\prime \prime} \mathrm{N}, 108^{\circ} 24^{\prime} 3.06^{\prime \prime E}, 102 \mathrm{~m}$ alt., in sugarcane rhizosphere, $11 \mathrm{Feb} .2011$, L. Xie, M8 (HMAS 247985). 

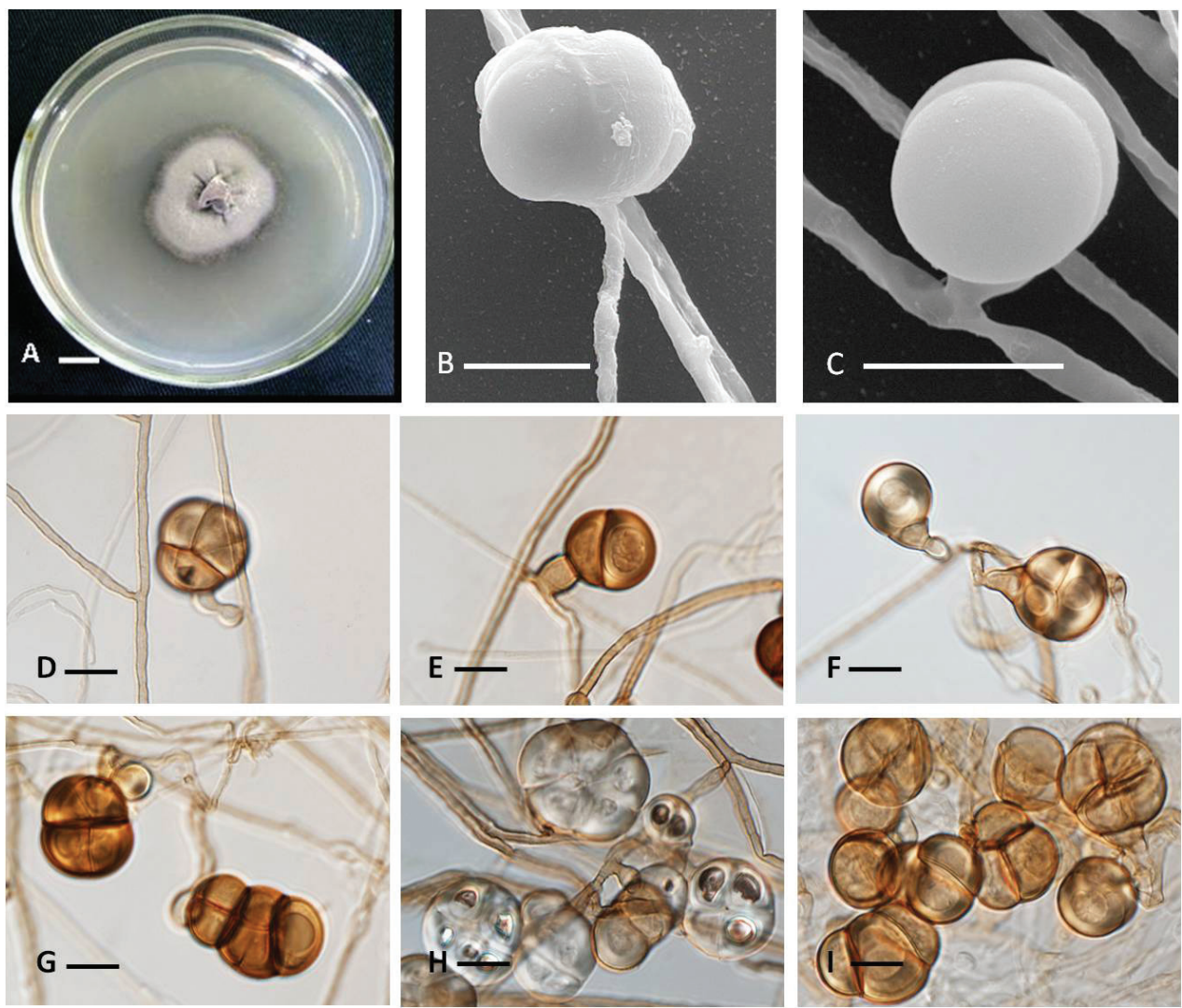

Figure I. The new species Conlarium nanningense (HMAS 247075, holotype). A Colony morphology B, C Scanning electron microscopy of conidia D-I Mature conidia. Scale bars: $10 \mathrm{~mm}(\mathbf{A}) ; 10 \mu \mathrm{m}(\mathbf{B}-\mathbf{E})$.

Notes. Conlarium nanningense is similar to the asexual morph of C. aquaticum, C. duplumascospora, and C. thailandense. They all have monoblastic, holoblastic conidiogenous cells and mostly irregular, brown, clathrate, muriform conidia (Liu et al. 2012). However, $C$. nanningense can be easily distinguished from $C$. aquaticum, $C$. duplumascospora, and $C$. thailandense by the number of conidial septa (2-4-transversely septate, 1-3-longitudinally septate in $C$. duplumascospora; 6-12-transverse septa, 4-10-longitudinal septa in C. aquaticum; 4-8-transverse septa, 4-6-longitudinal septa in $C$. thailandense vs $0-1$ transversely septa, $0-4$ longitudinal septa in $C$. nanningense) and conidial size $(15.5-35 \times 11-26.5 \mu \mathrm{m}$ in $C$. duplumascosporum, 45-70 $\times 20-57$ $\mu \mathrm{m}$ in $C$. aquaticum, 25-45 $\times 17-33 \mu \mathrm{m}$ in C. thailandense and $11-21 \times 9-21 \mu \mathrm{m}$ in C. nanningense) (Liu et al 2012; Zhang et al. 2017; Phookamsak et al. 2019). Phylogenetic reconstructions based on SSU+ITS+LSU+RBP2 sequences show authentic $C$. nanningense is sister to $C$. duplumascospora. A comparison of ITS pairwise indicates that $C$. nanningense differs from $C$. aquaticum, $C$. duplumascospora, and $C$. thailandense in $21 \mathrm{bp}, 12 \mathrm{bp}$, and $18 \mathrm{bp}$, respectively. Thus, following the guidelines of Jeewon and Hyde (2016), this is a new species. 


\section{Conlarium baiseense L.Xie, Y.L.Chen \& B.Liu, sp. nov.}

MycoBank: MB821682

Figure 2

Etymology. The species is named for Baise City, the type locality.

Type. CHINA. Guangxi: Baise City, Tiandong County, Silin Town. 2330'38"N, $107^{\circ} 20^{\prime} 1$ "E, $109 \mathrm{~m}$ alt., in sugarcane rhizosphere, 11 Sep 2015, Y.L. Chen and L.P. Qin, TD2 (HMAS 247298, holotype) deposited in Microbiology Research Institute, Guangxi Academy of Agricultural Science.

Description. Colony reached $14 \mathrm{~mm}$ diameter on medium after 2 weeks at $28^{\circ} \mathrm{C}$, grey-white to grey, circular, flat growth, less aerial hyphae, regular edge of colony. Hyphae light yellow-green to light yellow-brown, septate. Conidiophores yellow-brown, mostly stubby, 0-2-branched, 0-8-septate, straight or flexuous, 3-12 $\times$ 2-6 $\mu \mathrm{m}(7 \pm 2 \times 4 \pm 1 \mu \mathrm{m}, n=51)$. Conidiogenous cells determinate, doliiform, yellowbrown to brown, 3-8 $\times 5-12 \mu \mathrm{m}(6 \pm 1 \times 7 \pm 2 \mu \mathrm{m}, n=51)$. Muriform conidia yellow-brown to brown, irregularly globose or subglobose, smooth, constricted at the separation, $0-1$ transversely septa, $0-4$ longitudinal septa, $15-25 \times 12-19 \mu \mathrm{m}(18 \pm$ $2 \times 15 \pm 2 \mu \mathrm{m}, n=26)$. Columnar conidia, yellow-brown to brown, $2-5$ transversely septa, no longitudinal septa, $21-35 \times 7-12 \mu \mathrm{m}(28 \pm 5 \times 10 \pm 1 \mu \mathrm{m}, n=23)$. Sexual morph: undetermined.

Habitat and distribution. In sugarcane rhizosphere soil of southern China.

Other specimens examined. CHINA. Guangxi: Baise City, Tiandong County, Silin Town. $23^{\circ} 30^{\prime} 3.68^{\prime \prime} \mathrm{N}, 107^{\circ} 20^{\prime} 1 " \mathrm{E}, 112.5 \mathrm{~m}$ alt., in sugarcane rhizosphere, 11 Sep. 2015, Y.L. Chen and L.P. Qin, TD17 (HMAS 247986).

Notes. Conlarium baiseense is similar to the asexual morph of $C$. aquaticum, $C$. duplumascospora, C. nanningense, and $C$. thailandense. They all have monoblastic, holoblastic, conidiogenous cells and mostly irregular, brown, clathrate, muriform conidia (Liu et al. 2012). However, C. baiseense can be easily distinguished from C. aquaticum, C. duplumascospora, $C$. nanningense, and $C$. thailandense by its conidial septa number (6-12-transverse septa, 4-10-longitudinal septa in C. aquaticum; 2-4-transversely septate, 1-3-longitudinally septate in $C$. duplumascospora; $0-1$ transversely septa, $0-4$ longitudinal septa in $C$. nanningense; 4-8-transverse septa, 4-6-longitudinal septa in $C$. thailandense vs $0-2$ transversely septa, $0-8$ longitudinal septa in $C$. baiseense) and conidial size (15.5-35 $\times 11-26.5 \mu \mathrm{m}$ in $C$. duplumascosporum, $45-70 \times 20-57 \mu \mathrm{m}$ in $C$. aquaticum, 25-45 $\times 17-33 \mu \mathrm{m}$ in $C$. thailandense, $11-21 \times 9-21 \mu \mathrm{m}$ in $C$. nanningense vs $21 \times 35-7 \times 12 \mu \mathrm{m}$ in C. baiseense) (Liu et al 2012; Zhang et al. 2017; Phookamsak et al. 2019). Phylogenetic reconstructions based on SSU+ITS+LSU+RBP2 sequences shows that authentic $C$. baiseense form independent monophyletic groups, well separated from $C$. aquaticum, $C$. duplumascospora, $C$. nanningense, and $C$. thailandense, respectively. A comparison of ITS sequence shows that $C$. baiseense differs from $C$. aquaticum, $C$. duplumascospora, $C$. nanningense, and $C$. thailandense in $26 \mathrm{bp}, 24 \mathrm{bp}$, $18 \mathrm{bp}$, and $24 \mathrm{bp}$, respectively. According to the guidlines in Jeewon and Hyde (2016), we introduce $C$. nanningense as a new species. 

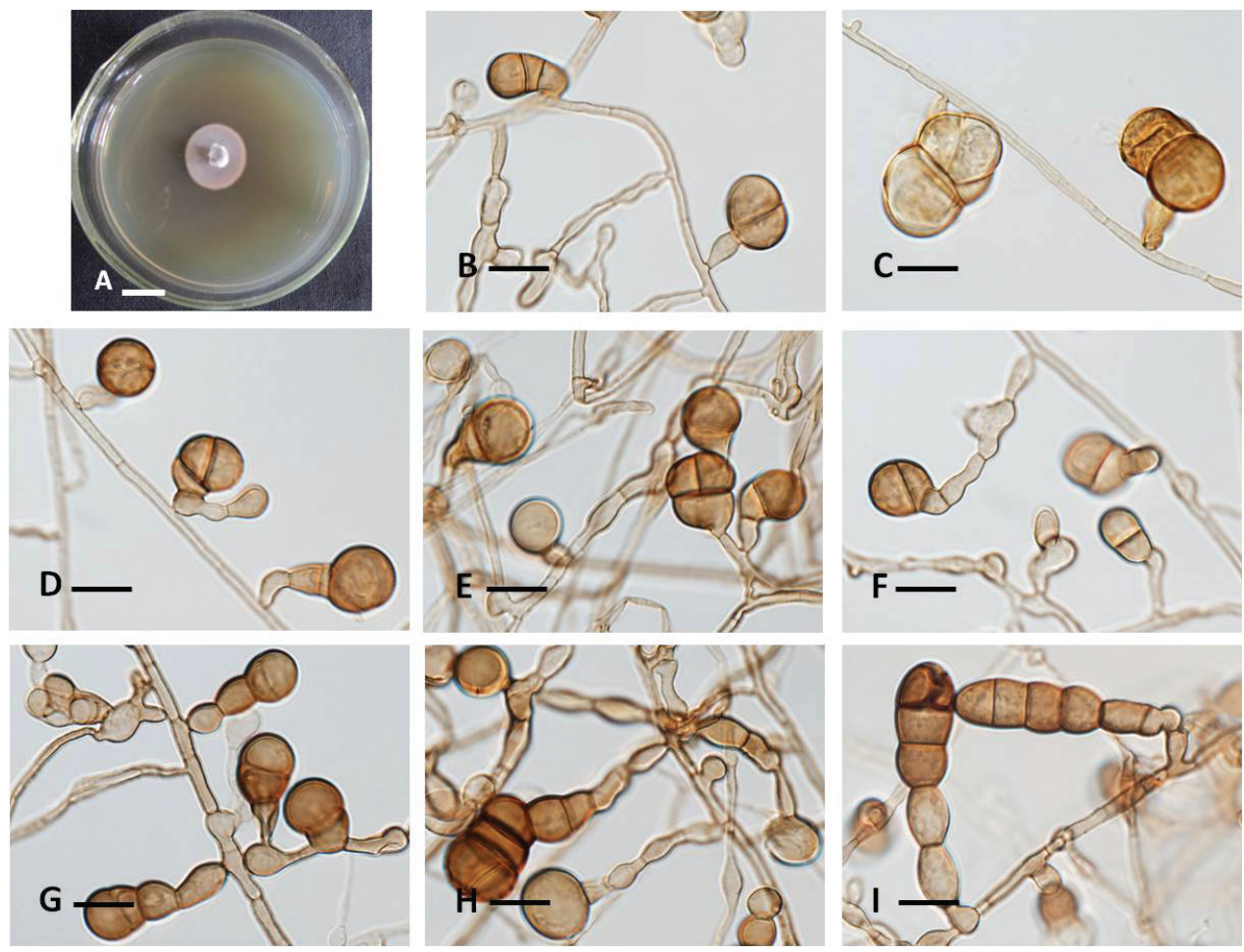

Figure 2. The new species Conlarium baiseense (HMAS 247298, holotype). A Colony morphology B-I Conidiophores, conidiogenous cells and conidia. Scale bars: $10 \mathrm{~mm}(\mathbf{A}) ; 10 \mu \mathrm{m}(\mathbf{B})$.

\section{Conlarium sacchari L.Xie, Y.L.Chen \& B.Liu, sp. nov.}

MycoBank: MB821681

Figure 3

Etymology. The epithet "sacchari" refers to the habitat where first collected.

Type. CHINA. Guangxi: Chongzuo City, Daxin County, Lanxu Village. $22^{\circ} 44^{\prime} 46^{\prime \prime N}, 107^{\circ} 15^{\prime} 15^{\prime \prime E}, 241 \mathrm{~m}$ alt., in sugarcane rhizosphere, 8 July 2015, Y.L. Chen and L.P. Qin, DX4 (HMAS 247299, holotype) deposited in Microbiology Research Institute, Guangxi Academy of Agricultural Science.

Description. Colony reached $15 \mathrm{~mm}$ diameter on medium after 2 weeks at $28^{\circ} \mathrm{C}$, greywhite to grey, circular, flat growth, less aerial hyphae, regular edge of colony. Hyphae light yellow to yellow-brown, septate. Conidiophores yellow-brown, mostly stubby, 0-2-branched, 0-6-septate, straight or flexuous, 3-30×2-4 $\mu \mathrm{m}(10 \pm 7 \times 3 \pm$ $1 \mu \mathrm{m}, n=43)$. Conidiogenous cells determinate, doliiform, yellow-brown to brown, $4-12 \times 2-7 \mu \mathrm{m}(7 \pm 2 \times 5 \pm 1 \mu \mathrm{m}, n=52)$. Conidia yellow-brown to brown, muriform, irregularly globose or subglobose, smooth, constricted at the separation, $0-1$ transversely septa, $0-4$ longitudinal septa, $14-19 \times 13-22 \mu \mathrm{m}(17 \pm 3 \times 16 \pm 2 \mu \mathrm{m}, n$ $=20)$. Sexual morph: undetermined. 

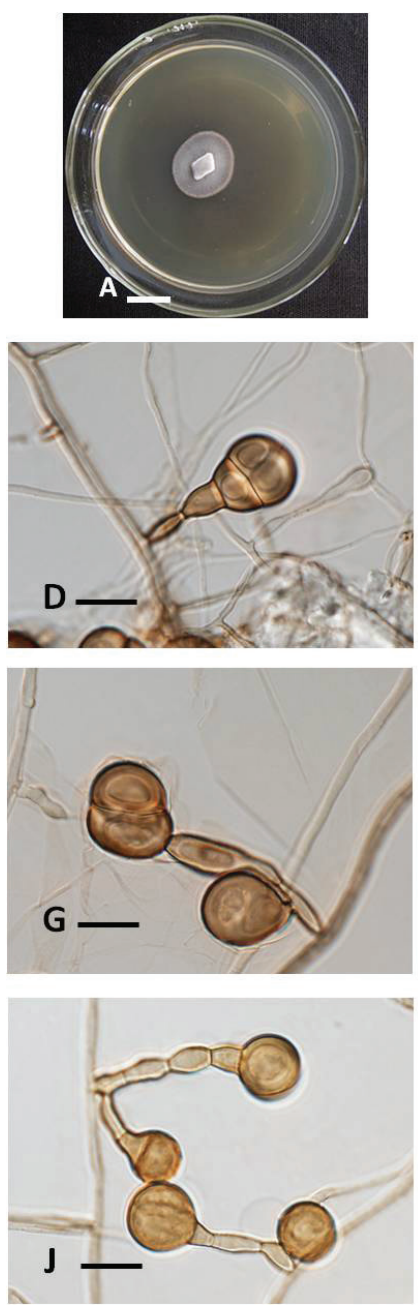
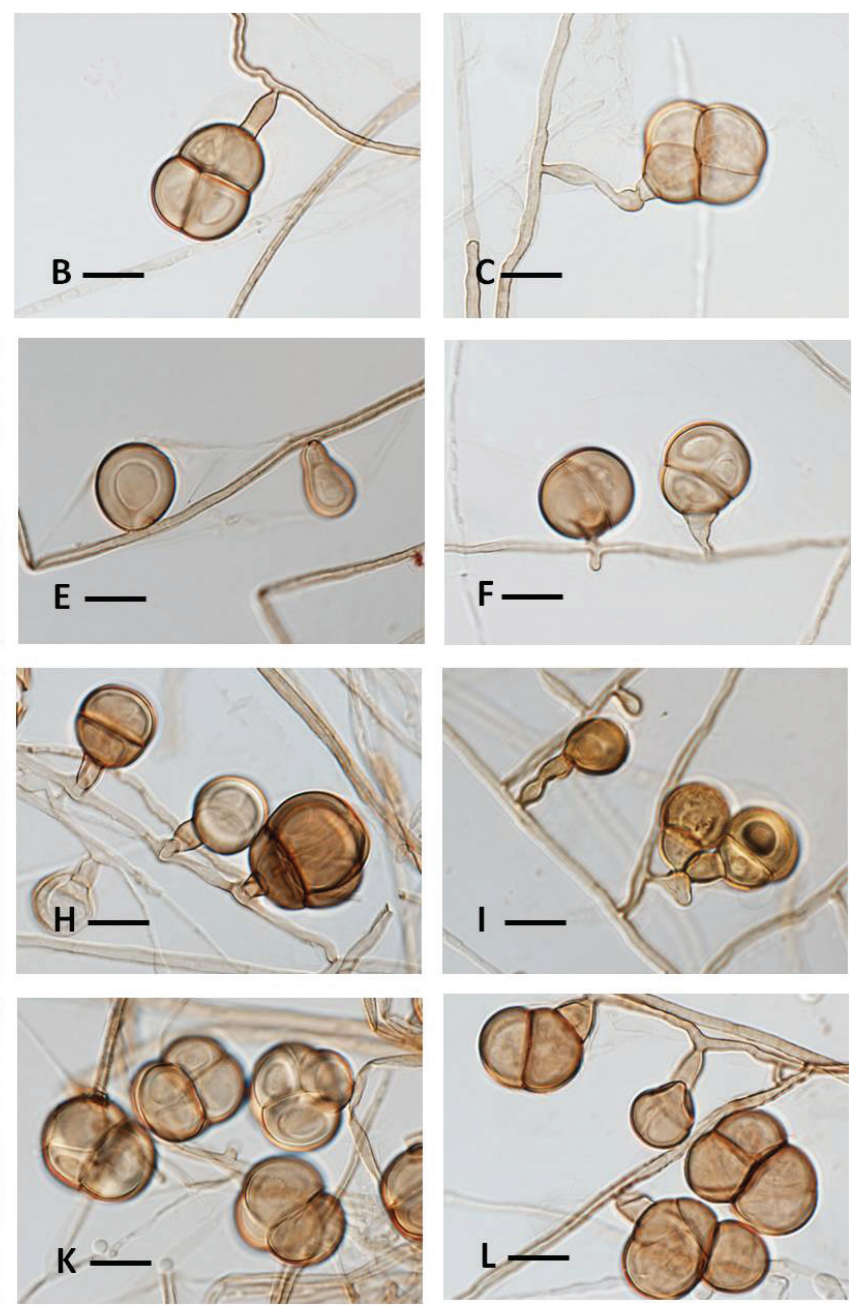

Figure 3. The new species Conlarium sacchari (holotype, HMAS 247299). A Colony morphology B-L Conidiophores, conidiogenous cells and conidia. Scale bars: $10 \mathrm{~mm}(\mathbf{A}) ; 10 \mu \mathrm{m}(\mathbf{B}-\mathbf{L})$.

Habitat and distribution. In sugarcane rhizosphere soil of southern China.

Other specimens examined. CHINA. Guangxi: Nanning City, Long'an County, Natong Town. $23^{\circ} 4^{\prime} 48^{\prime \prime} \mathrm{N}, 107^{\circ} 47^{\prime} 31^{\prime \prime E}, 128 \mathrm{~m}$ alt., in sugarcane rhizosphere, 11 Sep. 2015, Y.L. Chen and L.P. Qin, LA3 (HMAS 247300). Nanning City, Suxu town. $23^{\circ} 34^{\prime} 42^{\prime \prime N}, 108^{\circ} 8^{\prime} 30^{\prime \prime E}, 325 \mathrm{~m}$ alt., in sugarcane rhizosphere, 11 Feb. 2011, L. Xie, NN1 (HMAS 247301).

Notes. Conlarium sacchariis similar to the asexual morph of C. aquaticum, C. baiseense, C. duplumascospora, C. nanningense, and C. thailandense. They all have monoblastic, holoblastic, conidiogenous cells and mostly irregular, brown, clathrate, muriform conidia (Liu et al. 2012). However, Conlarium sacchari can be easily distinguished from $C$. aquaticum, $C$. duplumascospora, $C$. nanningense, and $C$. thailandense by its 
less number of conidial septa (6-12-transverse septa, 4-10-longitudinal septa in $C$. aquaticum; 2-5 transversely septa, 0-2 longitudinal septa in C. baiseense, 2-4-transversely septate, 1-3-longitudinally septate in $C$. duplumascospora; 0-1 transversely septa, 0-4 longitudinal septa in $C$. nanningense; 4-8-transverse septa, 4-6-longitudinal septa in C. thailandense vs. 0-1 transversely septa, 0-3 longitudinal septa in C. sacchari) (Liu et al 2012; Zhang et al. 2017; Phookamsak et al. 2019). Phylogenetic reconstructions based on SSU+ITS+LSU+RBP2 sequences shows that authentic C. sacchari formed independent monophyletic groups which are well separated from C. aquaticum, C. baiseense, C. duplumascospora, C. nanningense, and C. thailandense, respectively. A comparion of ITS sequence shows that $C$. sacchari differ from $C$. aquaticum, $C$. baiseense, C. duplumascospora, C. nanningense, and $C$. thailandense in $21 \mathrm{bp}, 24 \mathrm{bp}, 21$ bp, $18 \mathrm{bp}$, and $16 \mathrm{bp}$, resectively. Therefore, we introduce $C$. sacchari as a new species, following the guidelines of Jeewon and Hyde (2016).

\section{Phylogenetic analysis}

To determine the phylogenetic positions of the three new species, C. baiseense, $C$. nanningense and C. sacchari, all available SSU, ITS, LSU, and RBP2 sequences of Conlarium species and related genera in GenBank were downloaded (Table 1). A combined SSU+ITS+LSU+RBP2 dataset of $C$. baiseense, $C$. nanningense, and $C$. sac-

Table I. Taxa with GenBank accession numbers for SSU, ITS, LSU and RPB2.

\begin{tabular}{lccccc}
\hline \multicolumn{1}{c}{ Taxa } & Voucher & \multicolumn{4}{c}{ GenBank no. } \\
\cline { 2 - 6 } & & SSU & ITS & LSU & RPB2 \\
\hline Conlarium nanningense & M1 & KX886203 & KX886204 & KX886202 & MK224589 \\
Conlarium baiseense & TD2 & MF083159 & MF083157 & MF083158 & MK573000 \\
Conlarium sacchari & NN1 & MF083162 & MF083160 & MF083161 & MK224588 \\
Conlarium sacchari & LA3 & MF083165 & MF083163 & MF083164 & MK573001 \\
Conlarium sacchari & DX4 & MF083168 & MF083166 & MF083167 & MK224587 \\
Conlarium baiseense & TD17 & MK164657 & MK164653 & MK164655 & MK572999 \\
Conlarium nanningense & M8 & MK164658 & MK164654 & MK164656 & MK572998 \\
Conlarium duplumascospora & CGMCC 14938 & JN936987 & JN936995 & JN936991 & NS \\
Conlarium duplumascospora & CGMCC 14939 & JN936988 & JN936996 & JN936992 & NS \\
Conlarium duplumascospora & CGMCC 14940 & JN936989 & JN936997 & JN936993 & NS \\
Conlarium aquaticum & MFLUC 15-0992 & MF374372 & MF374354 & MF374363 & NS \\
Conlarium thailandense & MFLCC 17-2349 & MH624128 & MH624129 & MH624127 & NS \\
Atractospora thailandensis & KUMCC 16-0067 & MF374371 & MF374353 & MF374362 & MF370951 \\
Atractospora reticulata & CBS 127884 & NS & KT991669 & KT991660 & KT991649 \\
Atractospora reticulata & CBS 138740 & NS & KT991670 & KT991661 & KT991650 \\
Atractospora decumbens & CBS 139032 & KT991640 & KT991667 & KT991658 & KT991647 \\
Atractospora verruculosa & CBS 132040 & KT991641 & KT991668 & KT991659 & KT991648 \\
Pseudoproboscispora thailandensis & MFLUCC 15-0989 & MF374377 & MF374360 & MF374369 & NS \\
Rubellisphaeria abscondita & CBS 132078 & KT991646 & KT991678 & KT991666 & KT991657 \\
Lentomitella cirrhosa & ICMP 15131 & AY761089 & KY931780 & AY761085 & KM492911 \\
Torrentispora biatriispora & A 464-3 & NS & KY931803 & AY316352 & KY931858 \\
\hline Notes: NS No & & & &
\end{tabular}

Notes: NS No data in GenBank. 
chari, six isolates from Atractosporaceae, two taxa from Pseudoproboscisporaceae, and Lentomitella cirrhosa as the outgroup, were included in the phylogenetic analysis. In the alignment of the 21 sequences (SSU+ITS+LSU+RBP2), the data matrix comprised 3293 characters. The alignment dataset was performed using the MrBayes program, applied with SYM+I+G model selected by MrModeltest as the best-fit model. The prior probability density is a flat Dirichlet (all values are 278 1.0) for both Revmatpr and Statefreqpr as default settings. A Bayesian tree with posterior probability (BPP) and bootstrap values at branches is shown in Figure 4. In the phylogenetic tree, C. baiseense and C. sacchari formed a separate clade with 1.00 support of BPP and $100 \%$ support of NJ, C. nanningense formed a clade with $C$. duplumascospora with 0.96 support of BPP, and three of the new species were clearly separated from other Conlarium species.

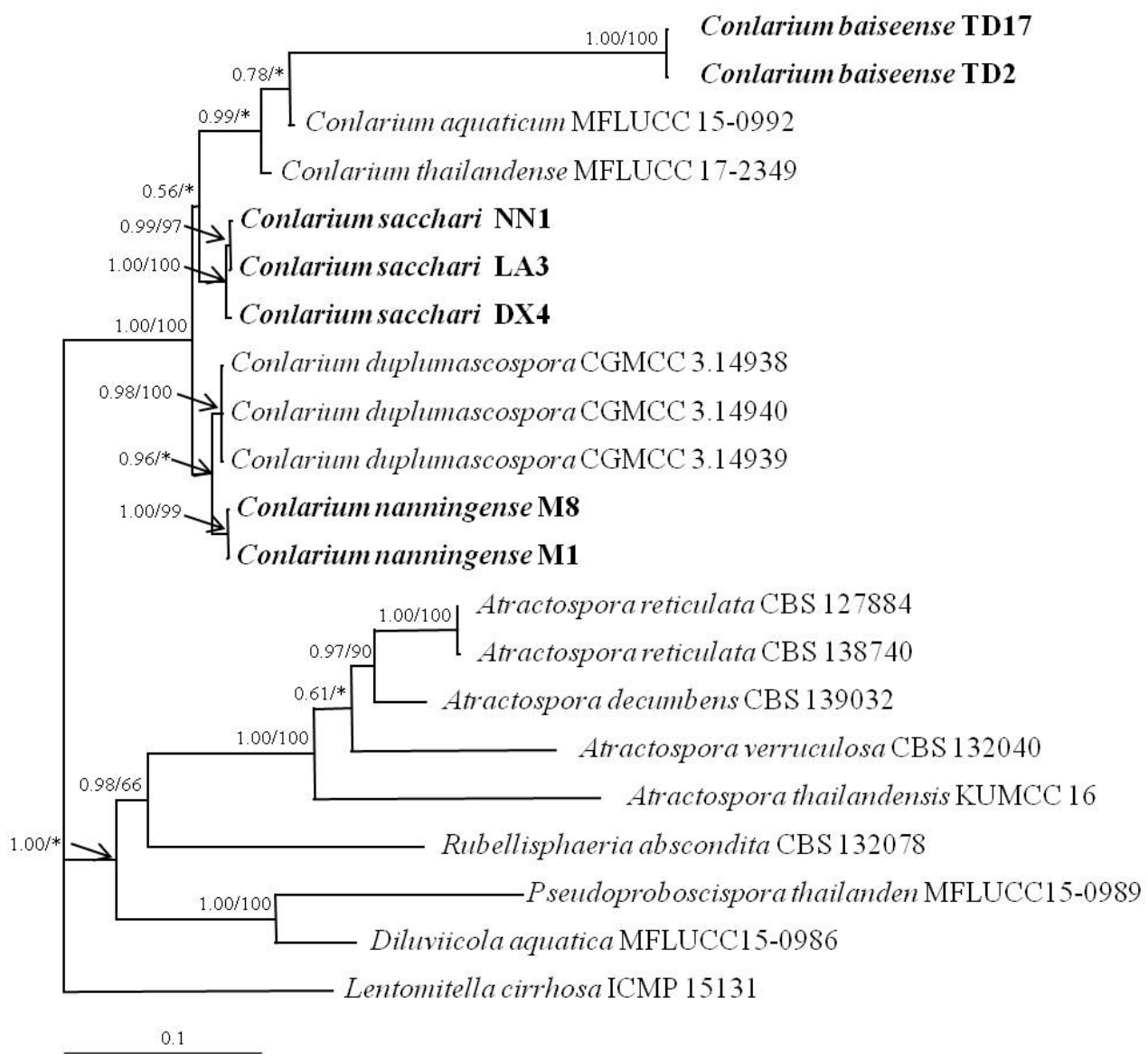

Figure 4. Bayesian tree based on the combined SSU+ITS+LSU+RBP2 sequences of Conlarium species and related families. Lentomitella cirrhosa was designated as outgroups. The numbers at each branch point represented Bayesian posterior probabilities (left) and percentage bootstrap support calculated from 1,000 replicates (right). ${ }^{*}$ indicates lack of support or support less than $50 \%$ for a particular clade. New species proposed are in bold. Bar 0.1 expected changes per site. 


\section{Discussion}

The genus Conlarium comprises three species, $C$. duplumascosporum, C. thailandense, and a hyphomycetous asexualmorph taxon, $C$. aquaticum. They have subglobose or irregular, brown, clathrate, muriform conidia (Zhang et al. 2017). The taxonomy of Conlarium is mainly based on the morphological characteristics of gregarious ascomata (Liu et al. 2012). However, the ascomata of C. aquaticum, C. thailandense, C. sacchari, $C$. baiseense, and $C$. nanningense was not observed on the medium. The new species introduced in this paper resemble the asexual morph of Conlarium in having muriform conidia. They can be distinguished by the fewer number of septa, as compared with C. duplumascosporum, C. aquaticum, and C. thailandense (Liu et al 2012; Zhang et al. 2017; Phookamsak et al. 2019). Conlarium baiseense can be distinguished from other species by its columnar conidia (more transverse and less longitudinal septa) present. Conlarium sacchari is characterized by simple conidia (fewer septa) and its longer branched conidiophore. Phylogenetic reconstructions based on SSU+ITS+LSU+RBP2 sequences show that the three new species form independent monophyletic groups and are well separated from $C$. duplumascophora, C. thailandense, and C. aquatcium; this further supports the erection of these three new species. Conlarium duplumascophora, $C$. thailandense, and $C$. aquatcium were from wood samples. Our three new species present as dark spetate endophytes from sugarcane rhizosphere and can be symbiotic with sugarcane. The new species extend the habitat of Conlarium from wood to soil.

\section{Acknowledgements}

This work was supported by China National Natural Science Foundation (No. 31460016), Guangxi Natural Science Foundation (No. 2015GXNSFBA139083), and Basic Scientific Research Special Project of Guangxi Academy of Agricultural Sciences (No. 2015YT80).

\section{References}

Huelsenbeck JP, Ronquist F (2001) MRBAYES: Bayesian inference of phylogeny trees. Bioinformatics 17: 754-755. https://doi.org/10.1093/bioinformatics/17.8.754

Jeewon R, Hyde KD (2016) Establishing species boundaries and new taxa among fungi: recommendations to resolve taxonomic ambiguities. Mycosphere 7 (11):1669-1677. https://doi. org/10.5943/mycosphere/7/11/4

Liu F, Hu DM, Cai L (2012) Conlarium duplumascospora gen. et. sp. nov. and Jobellisia guangdongensis sp. nov. from freshwater habitats in China. Mycologia 104: 1178-1186. https:// doi.org/10.3852/11-379

Liu YJ, Whelen S, Hall BD (1999) Phylogenetic relationships among ascomycetes: evidence from an RNA polymerse II subunit. Molecular Biology and Evolution 16: 1799-1808. https://doi.org/10.1093/oxfordjournals.molbev.a026092 
Narisawa K, Tokumasu S, Hashiba T (1998) Suppression of clubroot formation in Chinese cabbage by the root endophytic fungus, Heteroconium chaetospira. Plant Pathology 47: 206-210. https://doi.org/10.1046/j.1365-3059.1998.00225.x

Nylander JAA (2008) MrModeltest 2.3 REAME. http://www.abc.se/nylander/mrmodeltest2/ mrmodeltest2.html [Accessed 22 May 2008]

Phookamsak R, Hyde KD, Jeewon R, Bhat DJ, Jones EBG, Maharachchikumbura SSN, Raspé O, Karunarathna SC, Wanasinghe DN, Hongsanan S, Doilom M, Tennakoon DS, Machado AR, Firmino AL, Ghosh A, Karunarathna A, Mešić A, Dutta AK, Thongbai B, Devadatha B, Norphanphoun C, Senwanna C, Wei D, Pem D, Ackah FK, Wang GN, Jiang HB, Madrid H, Lee HB, Goonasekara ID, Manawasinghe IS, Kušan I, Cano J, Gené J, Li JF, Das K, Acharya K, Raj KNA, Latha KPD, KWT, He MQ, Dueňas M, Jadan M, Martín MP, Samarakoon MC, Dayarathne MC, Raza M, Park MS, Telleria MT, Chaiwan N, Mató́čec N, de Silva NI, Pereira OL, Singh PN, Manimohan P, Uniyal P, Shang QJ, Bhatt RP, Perera RH, Alvarenga RLM, Nogal-Prata S, Singh SK, Vadthanarat S, Oh SY, Huang SK, Rana S, Konta S, Paloi S, Jayasiri SC, Jeon SJ, Mehmood T, Gibertoni TB, Nguyen TTT, Singh U, Thiyagaraja V, Sarma VV, Dong W, Yu XD, Lu YZ, Lim YW, Chen Y, Tkalčec Z, Zhang ZF, Luo ZL, Daranagama DA, Thambugala KM, Tibpromma S, Camporesi E, Bulgakov TS, Dissanayake AJ, Senanayake IC, Dai DQ, Tang LZ, Khan S, Zhang H, Promputtha I, Cai L, Chomnunti P, Zhao RL, Lumyong S, Boonmee S, Wen TC, Mortimer PE, Xu JC (2019) Fungal diversity notes 929-1035: taxonomic and phylogenetic contributions on genera and species of fungi. Fungal Diversity 95: 1-273. https:// doi.org/10.1007/s13225-019-00421-w

Sun X, Guo LD (2010) Micronematobotrys, a new genus and its phylogenetic placement based on rDNA sequence analyses. Mycological Progress 9:567-574. https://doi.org/10.1007/ s11557-010-0664-7

Vilgalys R, Hester M (1990) Rapid genetic identification and mapping of enzymatically amplified ribosomal DNA from several Cryptococcus species. Journal of Bacteriology 172: 4238-4246. https://doi.org/10.1128/jb.172.8.4238-4246.1990

White TJ, Bruns T, Lee S, Taylor J (1990) Emplification and direct sequencing of fungal ribosomal RNA genes for phylogenetics. In: Innis MA, Gelfand DH, Sninsky JJ, White JT (Eds) PCR Protocols: a Guide to Methods and Applications. Academic Press, San Diego, California, 315-322. https://doi.org/10.1016/B978-0-12-372180-8.50042-1

Zhang H, Dong W, Hyde DK, Maharachchikumbura SSN, Hongsanan S, Bhat DJ, Al-Sadi AM, Zhang D (2017) Towards a natural classification of Annulatascaceae-like taxa: introducing Atractosporales ord. nov. and six new families. Fungal Diversity 85: 75-110. https://doi.org/10.1007/s13225-017-0387-z 